\title{
Validating the Family History Scoring System application to identify Lynch syndrome families
}

\author{
Ellen McGannon ${ }^{1 *}$, Janet Shenal ${ }^{1}$, Matthew A Strohhacker ${ }^{1}$, Susan Fay ${ }^{1}$, James M Church ${ }^{2}$ \\ From 14th Annual Meeting of the Collaborative Group of the Americas on Inherited Colorectal Cancer \\ Dallas, TX, USA. 12-13 October 2010
}

\section{Background}

The FHSS (a points-based scoring system) was previously used to identify families at high risk of an inherited colorectal cancer syndrome. AM-I (Amsterdam-I) families scored high $(\leq 12)$, but some scored low $(\geq 8)$ when scored from unaffected relative's perspective [1]. The FHSS is being applied to Lynch families $(\mathrm{MMR}+)$ and familial colon cancer type $\mathrm{X}$ (Type $\mathrm{X}$ ) to see if the scoring system would yield similar results.

\section{Method}

Family members (1 proband, 2 siblings and 2 children of the sibling) in Lynch and Type $\mathrm{X}$ families identified from the Jagelman Registry database were scored according to the score sheet described in Table 1 .

They were scored from perspective of an affected proband (AP) or unaffected proband (UP), affected (AS) or unaffected (US) sibling or a child of each sibling (Child).

\section{Results}

91 probands (68 affected, 23 unaffected) in 48 AM-I, 14 Amsterdam-II (AM-II), 6 Amsterdam-Like (AM-Like), 10 Familial Colon Cancer (FCC), 6 no syndrome (syn) families and 7 Type $\mathrm{X}$ were scored. 197 relatives were scored (38 affected siblings, 23 Child AP/AS, 40 Child AP/US, 77 unaffected siblings, 9 Child UP/AS, 10 Child UP/US). AM-I and Type X median scores were higher than other syndromes $(<12)$ and suggestive of HNPCC when scored from the perspective of the proband, sibling or child of AS. The median scores were lower in AM-II, FCC, AM-Like, and no syn families with fewer colon cancers (Table 2).

\section{Conclusion}

Findings were similar to validation study conducted on AM-I families. The FHSS is a reliable tool to determine familial risk of colorectal cancer especially in AM-I families and depends on the perspective of person being scored. In small families, those with predominantly extra colonic cancers or high-risk polyps, or if affected relatives are more than a generation from an individual, the FHHS will not always identify high risk or Lynch families.

\section{Author details}

${ }^{1}$ Taussig Cancer Institute, Cleveland Clinic, Cleveland, Ohio, USA.

${ }^{2}$ Department of Colorectal Surgery, The Sandford R. Weiss, M.D. Center for Hereditary Colorectal Neoplasia, Digestive Disease Institute, Cleveland Clinic, Cleveland, Ohio, 44195, USA.

Published: 10 March 2011

\section{Table 1}

\begin{tabular}{ll}
\hline & Points \\
\hline Proband & 3 \\
\hline Affected & \\
\hline First Degree Relatives(FDR) & 3 each \\
Each FDR & 3 \\
$1-$ FDR $<50-y r$, including proband) & Extra 3 \\
1-FDR-same side family & 1 each \\
\hline Second Degree relatives(SDR) & 1 \\
Each SDR & Extra $1^{*}$ \\
$>1-S D R<50-y r$ &
\end{tabular}

Combination FDR and SDR

1-FDR and any SDR-same side family

$2^{*}$

*only if maximum 3 points per family not exceeded.

* Correspondence: mcganne@ccf.org

${ }^{1}$ Taussig Cancer Institute, Cleveland Clinic, Cleveland, Ohio, USA

Full list of author information is available at the end of the article 
Table 2 Median Scores

\begin{tabular}{lllllllllll}
\hline & AP & AP's AS & AP's US & Child AP's AS & Child AP's US & UP & UP's AS & UP's US & Child UP's AS & Child UP's US \\
\hline Total Syndromes & 14 & 18 & 14 & 12 & 4 & 12 & 15 & 12 & 7 & 4 \\
AM- I & 17 & 18 & 17 & 12 & 5 & 12 & 16 & 12 & 9 & 4 \\
Type X & 14 & 17 & 14 & 14 & 4 & 12 & 15 & 12 & & \\
AM-II & 12 & 12 & 12 & 7 & 2 & 13 & 13 & 15 & 9 & 5 \\
FCC & 12 & 13 & 9 & 10 & 2 & 10 & 10 & 8 & 8 & 10 \\
AM-LIKE & 12 & & 12 & & 4 & 4 & & 4 & & 1 \\
No-Syn & 6 & & 6 & & 2 & 6 & 6 & & 6 & \\
\hline
\end{tabular}

\section{Reference}

1. Church JM: A scoring system for the strength of a family history of colorectal cancer. Dis Colon Rectum 2005, 48(5):889-96.

doi:10.1186/1897-4287-9-S1-P26

Cite this article as: McGannon et al:: Validating the Family History

Scoring System application to identify Lynch syndrome families.

Hereditary Cancer in Clinical Practice 2011 9(Suppl 1):P26.

Submit your next manuscript to BioMed Central and take full advantage of:

- Convenient online submission

- Thorough peer review

- No space constraints or color figure charges

- Immediate publication on acceptance

- Inclusion in PubMed, CAS, Scopus and Google Scholar

- Research which is freely available for redistribution

Submit your manuscript at www.biomedcentral.com/submit
C Biomed Central 